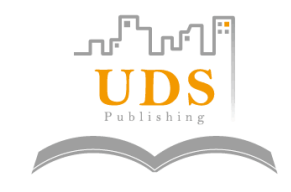

\title{
Application of Remote Sensing Information Technology and Geographic Information System in Land Dynamic Monitoring
}

\author{
Shaolu Zhang Zhenyu Feng Chengbing Huan \\ Huichuan District Natural Resources Information Technology Center, Zunyi, Guizhou, China
}

\begin{abstract}
In China, the protection and rational utilization of land resources is one of the basic national policies. Specifically, land resources occupy a very important position in the ecological environment system, and will also change because of the influence of natural geographical factors, human social activities and other factors. In order to protect and utilize land resources better, GIS and remote sensing information technology should be used reasonably in land dynamic monitoring, so as to achieve the expected effect of monitoring, predict the change of land resources and grasp the change trend of land resources. Based on this, this paper focuses on remote sensing information technology and GIS in land dynamic monitoring application for analysis for reference.
\end{abstract}

Keywords: Land dynamic monitoring; Land resources; Remote sensing information technology; Geographic information system

Citation: Shaolu Zhang, Zhenyu Feng, Chengbing Huan, 2020. Application of Remote Sensing Information Technology and Geographic Information System in Land Dynamic Monitoring. International Journal of Geology, 5(1): 12-15. http://doi. org/10.26789/IJG.2020.01.004

Copyright: Application of Remote Sensing Information Technology and Geographic Information System in Land Dynamic Monitoring. (C) 2020 Shaolu Zhang, Zhenyu Feng, Chengbing Huan. This is an Open Access article published by Urban Development Scientific Publishing Company. It is distributed under the terms of the Creative Commons Attribution-Noncommercial 4.0 International License, permitting all non-commercial use, distribution, and reproduction in any medium, provided the original work is properly cited and acknowledged.

\section{Introduction}

Although China's land area is very large, but the population is also very large, how to rationally plan land resources, and how to rationally use and plan land resources has become a major issue for China's future development. Therefore, in order to improve the efficiency of land resource utilization and realize the purpose of land resource protection, it is necessary to do a good job in land dynamic monitoring, rational use of remote sensing information technology and geographic information system, truly realize real-time monitoring of land resources, and further promote China The construction and development of the regional economy.

\section{Analysis of the Basic Content and Development Status of Remote Sensing Information Technology and Geographic Information System}

\subsection{Discovery Status Analysis of Remote Sensing Information Technology and Geographic Infor- mation System}

During the "Eighth Five-Year Plan" and "Ninth Five-
Year Plan" period, China has conducted in-depth research on geographic information systems and remote sensing information technology, accumulated very rich technical experience, and has been widely used nationwide. Nowadays, China has re-studied the land use and change in some key areas. Through the study of the driving conversion mechanism of land use, the use of land resources in China is carried out under the application of geographic information system and remote sensing information technology. Comprehensive analysis and analysis of the impact of changes in land resources on the ecological environment, so as to provide more comprehensive land resource data and information support for China's social and economic development.

\subsection{Basic Content of Remote Sensing Information Technology and Geographic Information System}

Both remote sensing information technology and geographic information system play a very important role in the development of land dynamic monitoring. The specific content is shown in Table 1. 
Application of Remote Sensing Information Technology and Geographic Information System in Land Dynamic Monitoring

Table 1. Basic content of remote sensing information technology and geographic information system

\begin{tabular}{|c|l|}
\hline Technology/System & \multicolumn{1}{c|}{ Fundamental contents } \\
\hline $\begin{array}{c}\text { Remote Sensing } \\
\text { Information Technology }\end{array}$ & $\begin{array}{l}\text { Remote sensing information technology is also called RS technology. Its principle is to use infrared and other high- } \\
\text { tech technologies to identify and detect long-distance substances, and judge the substances based on the reflection or } \\
\text { radiation function of the detected substance itself. Reasonable use of RS technology can provide a more detailed and } \\
\text { comprehensive understanding of the changes and use of land resources, and conduct dynamic monitoring of changes in } \\
\text { land resources. At the same time, remote sensing information technology can also record and analyze the actual situation } \\
\text { of land resources through the acquired dynamic data. }\end{array}$ \\
\hline Geographic Information & $\begin{array}{l}\text { Geographic information system is also called GIS system. This technology plays a very important role in land resource } \\
\text { information management. In the entire geographic information system, the advantages and functions of information } \\
\text { management can be maximized. Specifically, it can use spatial information technology to manage land resources to } \\
\text { ensure land resource design, land resource utilization, and land resource planning. Can get more accurate and reasonable } \\
\text { data information. At the same time, the GIS system can also be used to process and optimize the existing land resource } \\
\text { analysis data, to further optimize the design of the land resource model, and to use simulation experiments to improve the } \\
\text { accuracy of the results, which is useful for the dynamic monitoring of land resources. Information management provides } \\
\text { reliable technical support. }\end{array}$ \\
\hline
\end{tabular}

Nowadays, with the rapid improvement of the socio-economic level and the level of science and technology, remote sensing information technology has been widely used in the dynamic monitoring of land resources, thereby further improving the protection effect and utilization efficiency of land resources.

\section{Analysis of the Advantages of Remote Sensing Information Technology and Geographic Information System}

\subsection{Improve the Accuracy and Convenience of In- formation Acquisition}

In land resource management, the convenience and accuracy of data information acquisition are the basic and prerequisite conditions for carrying out land resource management, and it occupies a very important position in land resource management. Therefore, remote sensing information technology and geographic information systems should be used rationally, and multiple technologies should be combined to accurately locate land resources. At the same time, a fast, accurate, and macro-coverage method should be used ${ }^{[1]}$, and a comprehensive inspection through remote sensing information technology Obtain the land resource data information, and analyze and obtain evidence.

\subsection{Store a Large Amount of Information to Im- prove the Effect of Information Processing}

In the development of land resource management, the storage and processing of data information is also very important, and this is also the key point of spatial information technology. Reasonable use of GIS geographic information system can better store and detect the acquired information. At the same time, the data can be rented through the GIS geographic information system to achieve the purpose of spatial analysis and dynamic analysis of land resources, and further promote the development of these two tasks. In addition, in the management of land resources, GIS geographic information system can also be used to enable it to perform auxiliary and guiding functions, and to better store geographic information, so as to ensure that the acquired data is more accurate and to achieve correctness. The comprehensive analysis of data can comprehensively improve the protection effect and utilization efficiency of land resources.

\subsection{Improve the Effect of Data Analysis}

For land resources, it is very susceptible to human activities, changes in the natural environment and other factors. Therefore, the collected land resource data information should also change over time. Therefore, it should be focused Pay attention to the data and information generated by land resources in time and space. In order to have a comprehensive and detailed understanding of the information changes of land resources in time and space, high-tech space technology should be used to explore the changes of land resources, find the law of changes, and truly achieve the comprehensive treatment of land resources. Make a comprehensive analysis to prevent the occurrence of land resources and national land development, and avoid the loss of land resources. 


\subsection{Information Data Integrated System}

For the land resource information management in the land dynamic monitoring work, data information is the most important. Therefore, the acquired data should be properly stored and comprehensively analyzed. Using the data information system, through its data analysis and sorting capabilities and the ability to automatically generate reports, on the basis of time changes, the land resource information data can be sorted, searched, analyzed, and updated in time. Therefore, with the support of the information and data integrated system, it can provide more comprehensive functional support for solving such problems, improve the accuracy of data information, and ensure the efficiency of land resource management.

\section{Application Methods of Remote Sensing Information Technology and Geographic Information System in Land Dynamic Monitoring}

\subsection{Application of Remote Sensing Information Technology in Land Dynamic Monitoring}

Relevant land resource management departments use remote sensing information technology when carrying out land dynamic monitoring work to conduct a comprehensive analysis of the dynamic information technology generated in the process of land resource protection and utilization, and conduct systematic and comprehensive dynamics on it. It uses remote sensing information technology to sort and summarize various types of data information, establish a complete and dynamic data model, and then apply the model to construction projects, using relevant information and data calculation methods to save land resources The management department can better grasp the protection and utilization of land resources in the region, and conduct more in-depth and rigorous research on it.

For example, in our city, when conducting dynamic monitoring of land in a certain area, while using remote sensing satellite technology, it has carried out a number of data analysis and research work, such as SAR application data analysis and research, and land resource utilization. Evaluation research and so on.

\subsection{Application of Geographic Information System in Land Dynamic Monitoring}

The relevant practical results show that the geographic information system has very powerful spatial analysis capabilities. The system can automatically establish a complete data information database with its own capabilities, and conduct dynamic and real-time analysis of remote sensing image data. In this way, the acquired data can be analyzed and processed in time, and after that, based on the analysis and processing results, the spatial information decision of land resource use changes can be completed.

When carrying out land dynamic monitoring work, through the role of geographic information systems, relevant geospatial information can be analyzed. Specifically, it mainly includes analytical buffer areas, calculation of spatial data, and network analysis. At the same time, the The system can also add some specific content according to the user's personalized requirements. For example, a dynamic electronic data map of land resources can be formulated according to the requirements for use.

\subsection{Joint Application of Remote Sensing Information Technology and Geographic Information System in Land Dynamic Monitoring}

Nowadays, with the rapid improvement of China's scientific and technological level, both geographic information systems and remote sensing information technologies have been widely used in land resource management and have become the main technical tools in land dynamic monitoring. At the same time, with the in-depth study of these two technologies by scientific and technological researchers, the two technologies can be combined and applied with each other, and very good results have been achieved ${ }^{[2]}$.

On the one hand, the comprehensive use of geographic information system and remote sensing information technology can provide staff with more diversified and enriched information and data for land dynamic monitoring work, and further improve the accuracy of staff's analysis of information. The staff provides convenience for drawing drawings, and comprehensively improves the accuracy of drawing drawing and the pertinence of the drawing design plan.

On the other hand, the joint use of remote sensing information technology and geographic information system can make land dynamic monitoring work to be more automated, intelligent, informatized and centralized, and comprehensively improve the effect of land dynamic monitoring work and increase land resources. The utilization efficiency of the land resources can be used to achieve the purpose of protecting land resources.

\section{Conclusions}

In summary, in China's current land resource dynamic monitoring work, geographic information system and remote sensing information technology have become two very important technical tools. The rational use of the 
two technologies can improve the development of land resource dynamic monitoring work. Effectiveness, better grasp of land resource utilization and changes. Therefore, in-depth research should be conducted on these two technologies as the key development direction, so as to efficiently monitor and analyze the use and change of land resources, provide basic data support for land resource planning, and ensure land resources. Dynamic monitoring can be carried out smoothly.

\section{References}

[1] Wan Guobin, Hu Weiyang. Research on the Application of Remote Sensing Technology in the Field of Cadastral Surveying and Mapping[J]. Science and Technology Wind, 2019(23): 133.

[2] Yang Chao, Yao Qi. Research on the application of remote sensing images in land survey and dynamic monitoring[J]. China Metal Bulletin, 2019(07): $140+142$. 